Article

\title{
Astaxanthin Restrains Nitrative-Oxidative Peroxidation in Mitochondrial-Mimetic Liposomes: A Pre-Apoptosis Model
}

\author{
Camila M. Mano ${ }^{1,2,3}$, Thais Guaratini ${ }^{1,4}$ (D), Karina H. M. Cardozo ${ }^{1,5}$, Pio Colepicolo ${ }^{1}$, \\ Etelvino J. H. Bechara ${ }^{6,7}$ and Marcelo P. Barros ${ }^{2,8, * \text { (D) }}$ \\ 1 Departamento de Bioquímica, Instituto de Química, Universidade de São Paulo (IQUSP), \\ 05508-000 São Paulo, SP, Brazil; camilammano@gmail.com (C.M.M.); thais@lychnoflora.com.br (T.G.); \\ karina.cardozo@grupofleury.com.br (K.H.M.C.); piocolep@iq.usp.br (P.C.) \\ 2 Instituto de Ciências da Atividade Física e do Esporte (ICAFE), Universidade Cruzeiro do Sul, \\ 01506-000 São Paulo, SP, Brazil \\ 3 Superintendência da Polícia Técnico Científica, 05507-060 São Paulo, SP, Brazil \\ 4 Lychnoflora Pesquisa e Desenvolvimento em Produtos Naturais LTDA, 14030-090 Ribeirão Preto, SP, Brazil \\ 5 Grupo Fleury, 04344-070 São Paulo, SP, Brazil \\ 6 Departamento de Ciências Exatas e da Terra, Universidade Federal de São Paulo, Diadema, UNIFESP, \\ 09972-270 Diadema, SP, Brazil; ejhbechara@gmail.com \\ 7 Departamento de Química Fundamental, Instituto de Química, Universidade de São Paulo (IQUSP), \\ 05508-000 São Paulo, SP, Brazil \\ 8 Instituto de Agroquímica y Tecnología de Alimentos (IATA-CSIC), Departamento de Ciencia de los Alimentos, \\ Calle Catedrático Agustín Escardino 7, 46980 Paterna, Spain \\ * Correspondence: marcelo.barros@cruzeriodosul.edu.br or marcelobarros-iata@csic.es; \\ Tel.: +34-963-900-022 (ext. 2206)
}

Received: 22 February 2018; Accepted: 4 April 2018; Published: 12 April 2018

\begin{abstract}
Astaxanthin (ASTA) is a ketocarotenoid found in many marine organisms and that affords many benefits to human health. ASTA is particularly effective against radical-mediated lipid peroxidation, and recent findings hypothesize a "mitochondrial-targeted" action of ASTA in cells. Therefore, we examined the protective effects of ASTA against lipid peroxidation in zwitterionic phosphatidylcholine liposomes (PCLs) and anionic phosphatidylcholine: phosphatidylglycerol liposomes (PCPGLs), at different pHs (6.2 to 8.0), which were challenged by oxidizing/nitrating conditions that mimic the regular and preapoptotic redox environment of active mitochondria. Pre-apoptotic conditions were created by oxidized/nitr(osyl)ated cytochrome $c$ and resulted in the highest levels of lipoperoxidation in both PCL and PCPGLs (pH 7.4). ASTA was less protective at acidic conditions, especially in anionic PCPGLs. Our data demonstrated the ability of ASTA to hamper oxidative and nitrative events that lead to cytochrome c-peroxidase apoptosis and lipid peroxidation, although its efficiency changes with $\mathrm{pH}$ and lipid composition of membranes.
\end{abstract}

Keywords: mitochondria; membrane; lipid peroxidation; apoptosis; carotenoid; antioxidant; liposome; oxidative stress

\section{Introduction}

Dietary carotenoids are thought to provide health benefits by decreasing the risk of disease, particularly those in which free radical metabolism is a central mechanism of disease pathology. Accordingly, many carotenoids are powerful scavengers of reactive oxygen/nitrogen species (ROS/RNS) as well as quenchers of singlet oxygen, a highly reactive species towards proteins, lipids and nucleic 
acids [1]. Nonetheless, the redox chemistry of carotenoids is very complex at cellular loci and can drastically change under diverse microenvironmental conditions such as dielectric constant, polarity, $\mathrm{pH}$, and temperature. In fact, even prooxidant properties of carotenoids have been described under certain circumstances [2].

Astaxanthin (ASTA) is an orange-pink carotenoid abundant in marine organisms, including algae, zooplankton, mollusks, crustaceans, and salmon [3]. ASTA reportedly exhibits powerful in vitro and in vivo antioxidant and anti-inflammatory activities [4]. Regular or supplementary intake of ASTA in the diet has been shown to afford protection against some free radical-mediated disorders, such as ultraviolet radiation (UV)-promoted skin lesions and cancer, stomach ulcers caused by Helicobacter pylori infection, hepatocellular carcinoma, heart failure, macular degeneration, and prostate diseases [5].

Interestingly, a bulk of data supports a plausible mitochondrial-centered action of ASTA in isolated cells, tissues, and even living organisms, although an unequivocal mitochondria-targeted protective mechanism for ASTA has never been shown [6]. Our previous findings demonstrated that ASTA diminishes $\mathrm{Ca}^{2+}$ flow, as well as $\mathrm{NO}^{\bullet}, \mathrm{O}_{2}{ }^{\bullet-}$, and $\mathrm{H}_{2} \mathrm{O}_{2}$ production in isolated neutrophils and lymphocytes, which were then depicted as putative anti-apoptotic effects [7]. However, the current knowledge of the ASTA-apoptosis relationship is still unclear due to the use of different ASTA concentrations, cell types, and presence of other antioxidants or anti-inflammatory agents [8,9].

Concerning apoptotic mechanisms, oxidative/nitrative modifications of cytochrome c (cyt c) have been suggested as central molecular events underling the activation of caspase pathways in many cells undergoing apoptosis [10]. Peroxynitrite $\left(\mathrm{ONOO}^{-}\right)$is a powerful oxidizing/nitrating and nucleophilic agent that is generated by the diffusion-limited combination of $\mathrm{NO}^{\bullet}$ and $\mathrm{O}_{2}{ }^{\bullet-}$ radicals in cell. It is also a potent metabolite that modifies the structure and properties of cyt $\mathrm{c}$, thereby triggering apoptosis [11]. In addition to cardiolipin, phospholipids like phosphatidylglycerol (PG) can also change the affinity of both the native- and $\mathrm{ONOO}^{-}$-modified forms of cyt $\mathrm{c}$ in the inner mitochondrial membrane, thus contributing to the initiation of apoptosis [12].

As regular micronutrients in the human diet, carotenoids deserve more attention to clarify their complex chemistry in biological membranes, especially under different redox and $\mathrm{pH}$ conditions. To determine whether the antioxidant properties of ASTA are efficiently sustained under distinct redox conditions that mimic the mitochondrial environment, we exposed unilamellar zwitterionic egg yolk phosphatidyl choline (PCLs) and anionic phosphatidyl choline/phosphatidyl glycerol liposomes (PCPGLs) to oxidative/nitrative lesions that normally occur in mitochondria. In addition, an apoptosis-like condition was imposed by oxidized/nitr(osyl)ated cyt c at pHs ranging from 6.2 to 8.0. Our working hypothesis is that ASTA displays antioxidant properties that are modulated by the $\mathrm{pH}$ and lipid composition of membranes due to the structural effects of ASTA in terms of membrane fluidity/dynamics and/or to the association of cyt c (modified or not) with lipid bilayers under such conditions.

\section{Results}

\subsection{Oxidative/Nitrative Modifications on Cytochrome C}

The thermal decomposition of 3-morpholinosydnonimine (SIN-1) releases "free" superoxide $\left(\mathrm{O}_{2}{ }^{\bullet-}\right)$ and nitric oxide $\left(\mathrm{NO}^{\bullet}\right)$ radicals in air-equilibrated aqueous solution during a two-step process that uses the cationic sydnonimine intermediate SIN-1A ${ }^{\bullet+}$ and yields (4-morpholinylimino) acetonitrile (SIN-1C) as the final product (Figure S1A). None of these compounds absorbs visible light near the typical cyt $\mathrm{C}$ Soret band at $\lambda_{\max }=409 \mathrm{~nm}$, a wavelength peak that defines the redox behavior of cyt c (Figure S1B).

Free $\mathrm{NO}^{\bullet}$ in aqueous solution was identified by the fluorescent probe 4-amino-5-methylamino$2^{\prime}, 7^{\prime}$-difluorofluorescein (DAF-FM) in PCL at pH 7.4 (Figure 1). A similar DAF-FM kinetic pattern was observed in 10\% PCPGLs (Figure S2). The kinetics of NO ${ }^{\bullet}$ release was substantially hindered by the presence of both hemeproteins, cyt $\mathrm{c}$ and, for comparison, myoglobin $(\mathrm{Mb})$. Addition of superoxide dismutase (SOD), which totally consumes the $\mathrm{O}_{2}{ }^{\bullet-}$ radical, did not affect the identification of the $\mathrm{NO}^{\bullet}$ signal in aqueous solution. 


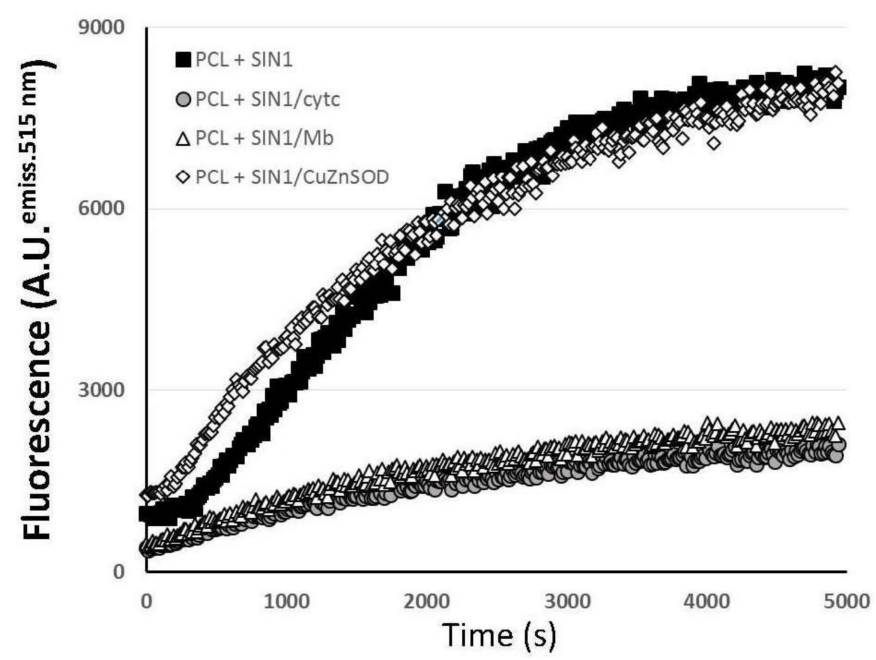

Figure 1. Fluorescence of 4-amino-5-methylamino- $2^{\prime}, 7^{\prime}$-difluorofluorescein (DAF-FM) with 3-morpholinosydnonimine (SIN-1) decomposition in $50 \mathrm{mM}$ phosphate buffer, $\mathrm{pH}$ 7.4, containing egg yolk phosphatidyl choline liposomes (PCL), hemeproteins (cytochrome c; cytc, or myoglobin; $\mathrm{Mb}$ ) or $\mathrm{Cu}, \mathrm{Zn}$-dependent superoxide dismutase (CuZnSOD).

Supplementary material 3 (Figure S3) shows the $150-\mu \mathrm{M}$ cyt c UV-Vis spectrum after a 45-min treatment with $150 \mu \mathrm{M} \mathrm{N}$-[4-[1-(3-aminopropyl)-2-hydroxy-2-nitrosohydrazino] butyl-1,3-propanediamine (spermine NONOate), an exclusive $\mathrm{NO}^{\bullet}$ donor, in aerated aqueous solution at $\mathrm{pH}$ 7.4. The coordination of $\mathrm{NO}^{\bullet}$ by the heme iron of ferricytochrome $\mathrm{c}$ can be observed by the appearance of a peak at $562 \mathrm{~nm}$. Expectedly, no changes were observed in the Soret band at $409 \mathrm{~nm}$ [13].

To detect structural heme pocket distortions induced by SIN-1 in cyt c (Soret band at $409 \mathrm{~nm}$ ), the absorbance was scanned at 10 and 20 min reaction times from 370 to $450 \mathrm{~nm}$. The calculated parameters of the spectrum smoothing procedure [fitting the Gaussian function, Equation (1)] are presented in Supplementary Materials 4 (Table S1), including Soret $\lambda_{\max }\left(\mathrm{x}_{\mathrm{c}}\right.$ parameter). Figure 2 summarizes the observed blue shift of the calculated cyt c Soret band at different SIN-1/cyt c ratios mixed with PCL in $50 \mathrm{mM}$ phosphate buffer, $\mathrm{pH}$ 7.4, which indicates the permanent acquisition of a peroxidase-like activity).

$\Delta \lambda_{\max }$

(Soret band)

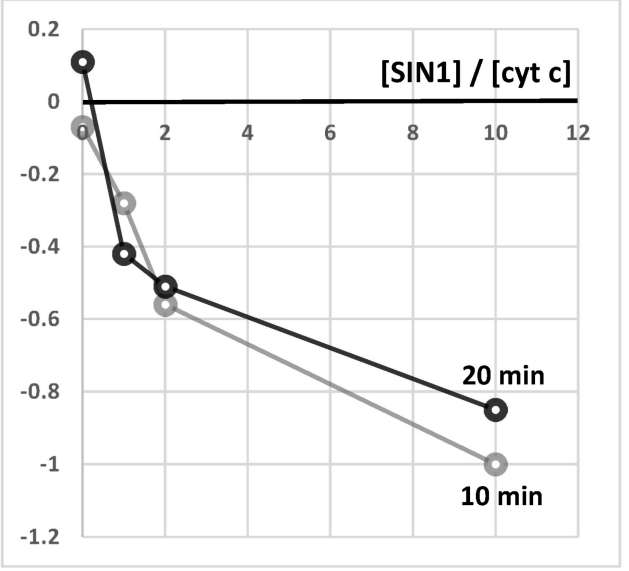

Figure 2. Shift of the cyt c Soret band $\left(\Delta \lambda_{\max }\right)$ treated with SIN-1 with PCL, in $50 \mathrm{mM}$ phosphate buffer, $\mathrm{pH} 7.4$, for $20 \mathrm{~min}$. 


\subsection{Peroxidation of Liposomes by the SIN-1/Cyt C System}

In comparison with untreated liposomes (control, C), both cyt $\mathrm{c}$ and SIN-1/cyt c resulted in a nine-fold increase in lipoperoxidation, expressed as the concentration of thiobarbituric acid-reactive substances (TBARS) in PCL (Figure 3). Similar increase was also observed when 10\% PCPGL was treated with SIN-1/cyt c, however lipid peroxidation was reduced by $25 \%$ when cyt $\mathrm{c}$ alone was used to oxidize these liposomes.

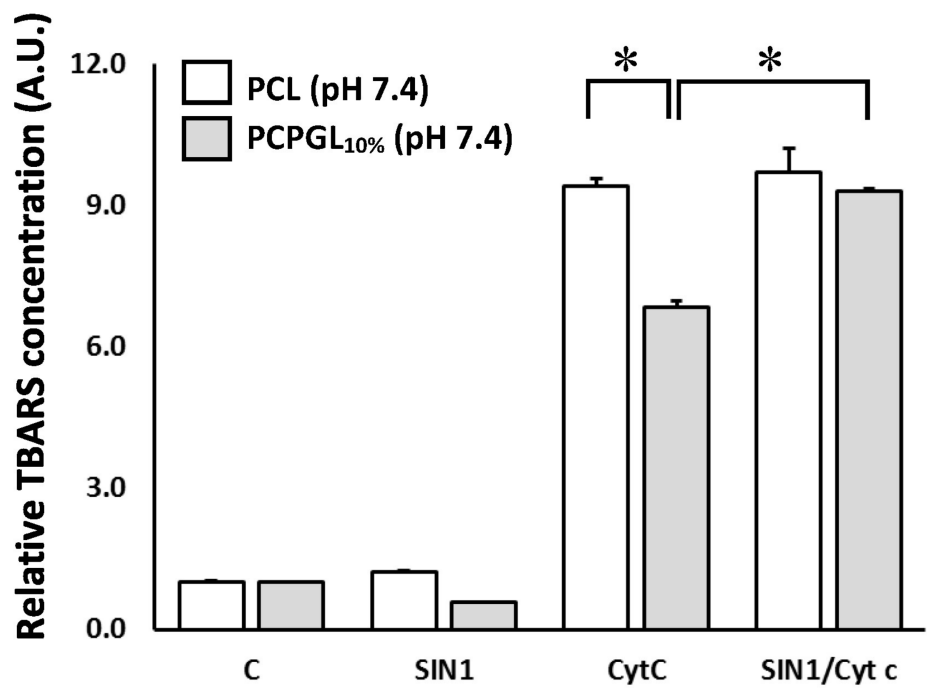

Figure 3. Thiobarbituric acid-reactive substances (TBARS) levels of PCL or phosphatidyl choline:phosphatidyl glycerol liposomes (PCPGLs) treated with cyt c, and/or SIN-1, at pH 7.4. $\left({ }^{*} p \leq 0.05\right)$.

\subsection{Astaxanthin Effect on Liposome Size}

As shown in Table 1, all liposomes showed equal diameters within experimental error both in the presence and absence of ASTA (Dynamic Light Scattering data).

Table 1. Average diameters of PCLs or PCPGLs in $50 \mathrm{mM}$ phosphate buffer, $\mathrm{pH} 7.4$, with/without $25 \mu \mathrm{M}$ ASTA $(n=3)$.

\begin{tabular}{ccc}
\hline Liposome & Control (Diameter, $\mathbf{n m})$ & $\mathbf{+ 2 5} \boldsymbol{\mu}$ M ASTA (Diameter, $\mathbf{n m}$ ) \\
\hline PCL & $196.7 \pm 0.8$ & $184.2 \pm 0.6$ \\
5\% PCPGL & $191.5 \pm 0.3$ & $181.2 \pm 0.3$ \\
10\% PCPGL & $189.1 \pm 1.3$ & $190.4 \pm 0.4$ \\
\hline
\end{tabular}

\section{4. $p H$ Effect on Lipid Peroxidation and Astaxanthin Antioxidant Properties}

Thiobarbituric acid-reactive substances levels were also measured in PCL and 10\% PCPGLs oxidized by SIN-1/cyt c in $50 \mathrm{mM}$ phosphate buffer at different pHs. Using non-oxidized liposomes as a reference (control in Table 2), the data depicted by Figure 4 show that lipid peroxidation of PCL was lower in acidic conditions compared to $\mathrm{pHs} 7.4$ and 8.0. These oxidative conditions were counteracted by $25 \mu \mathrm{M}$ ASTA, which provided maximal protection to membranes at $\mathrm{pH} 7.4(80 \%)$, whereas TBARS inhibition at $\mathrm{pH} 8.0$ was around $65 \%$. ASTA slightly inhibited lipid peroxidation in PCL at pHs $6.2(-20 \%)$ and $6.8(-30 \%)$. In anionic PCPGLs, TBARS levels at pHs 6.2 and 6.8 were almost two-fold higher than in PCL. No differences were observed between TBARS levels in PCL and $10 \%$ PCPGLs at pHs 7.4 and 8.0. In summary, ASTA exhibited no antioxidant effects in 10\% PCPGLs at acidic conditions, but only at $\mathrm{pHs} 7.4(-44 \%)$ and $8.0(-65 \%)$, offering less antioxidant protection than that observed in PCL at the same pHs. The effect of ASTA against lipoperoxidation induced by 
other mitochondrial-mimicking oxidative/nitrative conditions in PCL and 10\% PCPGL (see Materials and Methods, Section 4.5. Oxidative/Nitrative Conditions for Liposome Peroxidation), is presented in Table 2.

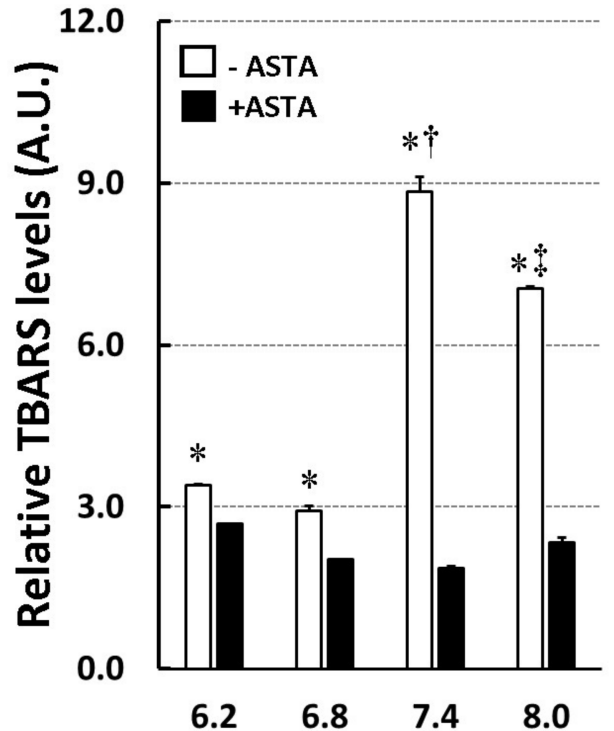

(a)

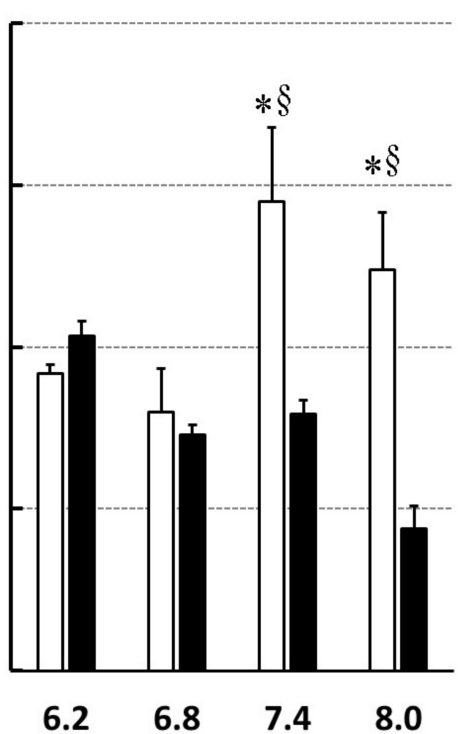

(b)

Figure 4. TBARS levels of (a) PCL or (b) PCPGLs, loaded (+ASTA) or not (-ASTA) with $25 \mu \mathrm{M}$ Astaxanthin (ASTA) and treated with SIN-1/cyt.c in $50 \mathrm{mM}$ phosphate buffer, in different $\mathrm{pH} .{ }^{*} p \leq 0.05$ compared to respective ASTA-loaded liposome; $\S p \leq 0.05$ compared to $\mathrm{pH} 6.2$ and $6.8 ;+p \leq 0.05$ compared to $\mathrm{pH} 6.2,6.8$, and 8.0; and $\ddagger p \leq 0.05$ compared to 6.2, 6.8, and 7.4.

Table 2. TBARS levels of PCL or PCPGLs treated with SIN-1, $\mathrm{KO}_{2}$, and/or SOD, in $50 \mathrm{mM}$ phosphate buffer, in different $\mathrm{pH}\left({ }^{*} p \leq 0.05\right)$.

\begin{tabular}{|c|c|c|c|c|c|c|c|c|c|}
\hline \multirow{3}{*}{\multicolumn{2}{|c|}{ TBARS Levels }} & \multicolumn{8}{|c|}{$\mathrm{pH}$} \\
\hline & & \multicolumn{2}{|c|}{6.2} & \multicolumn{2}{|c|}{6.8} & \multicolumn{2}{|c|}{7.4} & \multicolumn{2}{|c|}{8.0} \\
\hline & & -ASTA & +ASTA & -ASTA & +ASTA & -ASTA & +ASTA & -ASTA & +ASTA \\
\hline \multirow{3}{*}{ PCL } & SIN1 & $0.77 \pm 0.01$ & $1.19 \pm 0.01$ & $0.88 \pm 0.02$ & $0.95 \pm 0.01$ & $0.82 \pm 0.04$ & $1.10 \pm 0.02$ & $1.14 \pm 0.03$ & $1.02 \pm 0.03$ \\
\hline & $\mathrm{KO}_{2}$ & $0.79 \pm 0.05$ & $1.03 \pm 0.03$ & $0.77 \pm 0.22$ & $0.92 \pm 0.04$ & $1.06 \pm 0.07$ & $1.04 \pm 0.01$ & $0.98 \pm 0.05$ & $0.97 \pm 0.02$ \\
\hline & SIN1+SOD & $0.92 \pm 0.01$ & $1.18 \pm 0.03$ & $0.90 \pm 0.03$ & $0.95 \pm 0.07$ & $1.09 \pm 0.05$ & $1.15 \pm 0.02$ & $1.26 \pm 0.03$ & $1.06 \pm 0.03$ \\
\hline \multirow{4}{*}{ PCPGL 10\% } & SIN1 & $1.56 \pm 0.79$ & $1.21 \pm 0.20$ & $1.24 \pm 0.47$ & $1.35 \pm 1.00$ & $1.38 \pm 0.01$ & $0.88 \pm 0.01$ & $1.14 \pm 0.01$ & $0.90 \pm 0.04$ \\
\hline & $\mathrm{KO}_{2}$ & $2.25 \pm 0.49$ & $1.32 \pm 0.28$ & $1.16 \pm 0.26$ & $1.93 \pm 0.49$ & $1.10 \pm 0.05$ & $0.95 \pm 0.02$ & $0.95 \pm 0.04$ & $0.89 \pm 0.02$ \\
\hline & SIN1+SOD & $1.91 \pm 0.60$ & $1.11 \pm 0.26$ & $1.09 \pm 0.65$ & $0.86 \pm 0.37$ & $1.53 \pm 0.01$ & $0.90 \pm 0.03$ & $1.13 \pm 0.01$ & $0.92 \pm 0.01$ \\
\hline & $\mathrm{KO}_{2}+\mathrm{SOD}$ & $1.48 \pm 0.40$ & $2.21 \pm 0.25$ & $1.49 \pm 0.47$ & $1.30 \pm 0.35$ & $1.29 \pm 0.02$ & $0.97 \pm 0.02$ & $1.07 \pm 0.03$ & $0.92 \pm 0.02$ \\
\hline
\end{tabular}

The peroxidation data monitored by malondialdehyde (MDA) concentrations in PCL and 10\% PCPGLs (Figure 5) were comparatively similar to those observed through the TBARS assay in Figure 4. Accordingly, $25 \mu \mathrm{M}$ ASTA did not show any significant inhibition of lipid peroxidation measured as MDA levels at acidic conditions in either PCL or 10\% PCPGLs. Significant antioxidant activity of ASTA was therefore maximal at pH 7.4 in both PCL (-45\%) and 10\% PCPGLs (-37\%). At $\mathrm{pH}$ 8.0, ASTA inhibited MDA-lipid peroxidation by $24 \%$ in PCL and $15 \%$ in 10\% PCPGLs (Figure 5). The effect of $25 \mu \mathrm{M}$ ASTA against MDA-HPLC formation induced by other mitochondrial-mimicking oxidative/nitrative conditions in PCL and 10\% PCPGL (Section 4.5. Oxidative/Nitrative Conditions for Liposome Peroxidation), is presented in Table 3. 


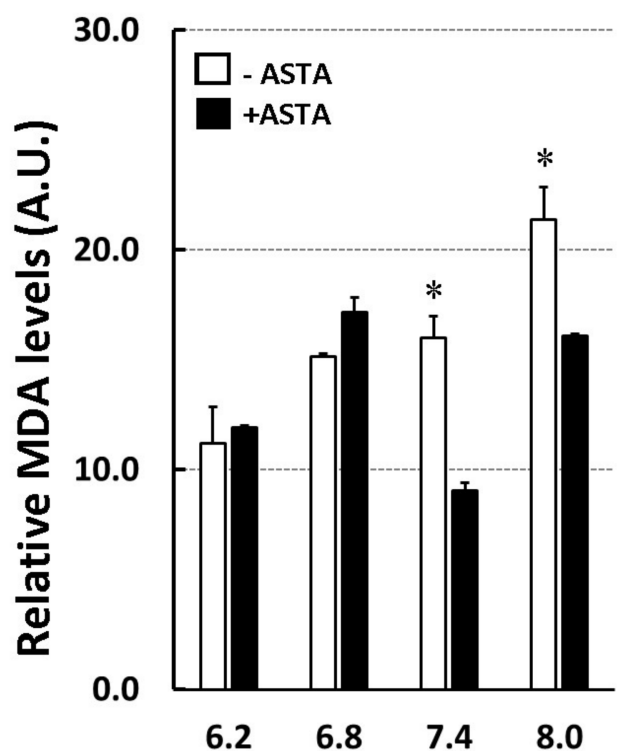

(a)

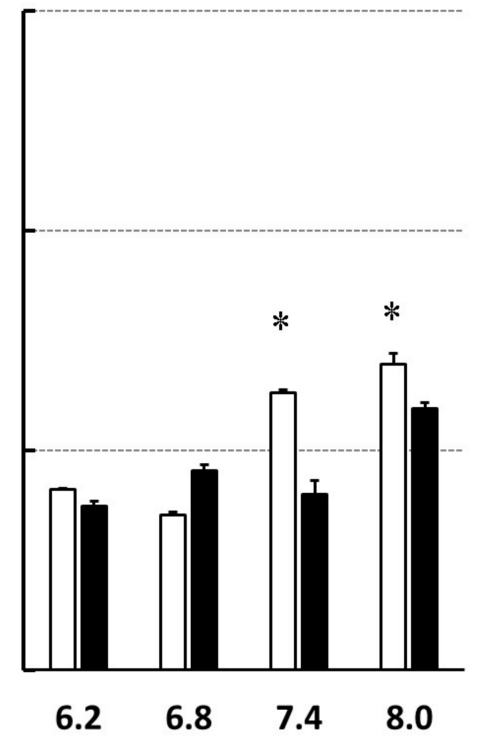

(b)

Figure 5. MDA levels of (a) PCL or (b) PCPGLs treated with SIN-1/cyt.c, in the presence or absence of $25 \mu \mathrm{M}$ ASTA, in $50 \mathrm{mM}$ phosphate buffer, in different $\mathrm{pH} .{ }^{*} p \leq 0.05$.

Table 3. MDA levels of PCL or PCPGLs treated with SIN-1, $\mathrm{KO}_{2}$, and/or SOD, in $50 \mathrm{mM}$ phosphate buffer, in different $\mathrm{pH}\left({ }^{*} p \leq 0.05\right)$.

\begin{tabular}{|c|c|c|c|c|c|c|c|c|c|}
\hline \multirow{3}{*}{\multicolumn{2}{|c|}{ MDA-HPLC Levels }} & \multicolumn{8}{|c|}{$\mathrm{pH}$} \\
\hline & & \multicolumn{2}{|c|}{6.2} & \multicolumn{2}{|c|}{6.8} & \multicolumn{2}{|c|}{7.4} & \multicolumn{2}{|c|}{8.0} \\
\hline & & -ASTA & +ASTA & -ASTA & +ASTA & -ASTA & +ASTA & -ASTA & +ASTA \\
\hline \multirow{3}{*}{ PCL } & SIN1 & $1.00 \pm 0.03$ & $1.37 \pm 0.05$ & $1.37 \pm 0.20$ & $1.78 \pm 0.06$ & $0.65 \pm 0.02$ & $0.84 \pm 0.02$ & $1.51 \pm 0.01$ & $1.72 \pm 0.01$ \\
\hline & $\mathrm{KO}_{2}$ & $1.17 \pm 0.03$ & i.d. & $1.29 \pm 0.30$ & i.d. & $1.28 \pm 0.01$ & $1.01 \pm 0.01$ & $1.10 \pm 0.16$ & $1.63 \pm 0.01$ \\
\hline & SIN1+SOD & $1.13 \pm 0.08$ & $1.35 \pm 0.07$ & $1.44 \pm 0.19$ & $1.70 \pm 0.01$ & $0.75 \pm 0.01$ & $0.87 \pm 0.02$ & $1.92 \pm 0.10$ & $1.34 \pm 0.01$ \\
\hline \multirow{4}{*}{ PCPGL 10\% } & SIN1 & $1.06 \pm 0.18$ & $1.27 \pm 0.09$ & $1.07 \pm 0.09$ & $1.08 \pm 0.09$ & $1.09 \pm 0.13$ & $1.69 \pm 0.06$ & $0.55 \pm 0.01$ & $1.35 \pm 0.14$ \\
\hline & $\mathrm{KO}_{2}$ & $1.06 \pm 0.13$ & $1.24 \pm 0.26$ & $1.07 \pm 0.11$ & $1.01 \pm 0.14$ & $1.13 \pm 0.14$ & $1.14 \pm 0.04$ & $0.97 \pm 0.06$ & $1.07 \pm 0.02$ \\
\hline & SIN1+SOD & $1.17 \pm 0.18$ & $1.18 \pm 0.06$ & $1.08 \pm 0.09$ & $1.19 \pm 0.08$ & $1.04 \pm 0.12$ & $1.00 \pm 0.14$ & $1.13 \pm 0.01$ & $1.07 \pm 0.14$ \\
\hline & $\mathrm{KO}_{2}+\mathrm{SOD}$ & $1.20 \pm 0.05$ & $1.20 \pm 0.14$ & $1.24 \pm 0.10$ & $1.21 \pm 0.15$ & $1.00 \pm 0.10$ & $1.02 \pm 0.06$ & $1.07 \pm 0.17$ & $1.00 \pm 0.41$ \\
\hline
\end{tabular}

i.d. $=$ inconsistent data.

\section{Discussion}

\subsection{Cytochrome c-Modified by SIN-1 Induces Liposome Peroxidation}

We aimed here to unveil the antioxidant properties of ASTA in a redox environment similar to that found in active mitochondria, including that linked to the initiation of apoptosis via cyt $\mathrm{c}$ oxidation/nitr(osyl)ation [14]. The combination of ROS/RNS such as $\mathrm{O}_{2}{ }^{\bullet-}, \mathrm{NO}^{\bullet}$, and $\mathrm{ONOO}^{-}$is known to change the redox behavior of cyt $\mathrm{c}$ in mitochondria, specifically its peroxidase activity and increased permeability via high valence heme intermediates [11]. Importantly, recent evidence shows that ASTA possibly targets the mitochondria in many animal cell models under a wide variety of physiological conditions [15].

First, redox alterations of cyt $\mathrm{c}$ upon treatment with SIN-1, a synthetic compound that decomposes into $\mathrm{O}_{2}{ }^{\bullet-}$ and $\mathrm{NO}^{\bullet}$ in aqueous solution [16] was revisited at planned experimental conditions. Our main findings are as follows: (i) $\mathrm{NO}^{\bullet}$ formed from SIN-1 decomposition interacts with heme group of cyt c (as well as myoglobin, for comparison) independently upon the presence of $\mathrm{O}_{2}{ }^{\bullet-}$ (Figure 1 and Figure S2); (ii) structural distortions in the vicinity of the heme group of cyt c are induced by 
SIN-1 treatment (Table S4 and Figure 2), which brings about redox changes of the protein properties (Figure 3); (iii) the cyt c misshapes were not caused by NO• alone (Figure S3) as suggested by the lack of effect in the presence of spermidine NONOate; instead, protein changes were only observed in the presence of SIN-1-formed $\mathrm{O}_{2}{ }^{--}$and $\mathrm{NO}^{\bullet}$; (iv) SIN-1 treatment of cyt c triggers the highest oxidizing conditions for all liposome systems (Figure 3); and (v) most importantly, the extent of lipid peroxidation is roughly identical in zwitterionic PCL or anionic 10\% PCPGLs after SIN-1/cyt c treatment (Figure 3). Although extensively studied, the mechanism of cyt c-catalyzed peroxidation of membrane phospholipids is not yet well understood [17]. Previous work has shown that $\mathrm{ONOO}^{-}$is not likely to drive cyt c modifications under the experimental conditions used here, since SIN-1-treated cyt $\mathrm{c}$ does not exhibit the spectral characteristics of nitrated cyt $\mathrm{c}$ produced by direct reaction with $\mathrm{ONOO}^{-}$[18].

The TBARS data shown in Figure 3 reveal that cyt $\mathrm{c}$ alone can catalyze lipid peroxidation in zwitterionic PCL at pH 7.4. Additional treatment with SIN-1 does not exacerbate the cyt c-induced lipid peroxidation process induced in these PCL, suggesting that SIN-1 does not fully or concomitantly interact with cyt c. Or else, it indicates that SIN-1-derived ROS/RNS does not impose further structural distortions in cyt c. Interestingly, TBARS levels were 35\% lower in 10\% PCPGLs compared to PCL treated with cyt c alone (Figure 3). It is expected that the cationic heme protein cyt c, (pI $\sim 10.4$, $\mathrm{pH}$ 7.4) has stronger electrostatic interactions with anionic membranes of PCPGLs. Nevertheless, such protein-lipid interactions were apparently insufficient to initiate lipid peroxidation compared to that observed in PCL. The presence of water-soluble lipid hydroperoxides formed during the preparation of liposomes could also possibly initiate the cyt c catalytic cycle under our experimental conditions here. Further experiments are necessary to investigate this hypothesis.

\subsection{Liposome Size/Organization is Unaltered by Astaxanthin Incorporation}

Liposome sizes were not affected by lipid composition or the association of ASTA with PCL or $10 \%$ PCPGLs (Table 1). Available data show that the incorporation of phosphatidylglycerol (PG) into PCL (of different compositions) does not affect liposome diameter/size, within the $\mathrm{pH}$ range studied here [19].

With respect to accessibility of ROS/RNS in liposomal membranes, the unsaturation index of phosphatidylcholine (PC) or PG was shown to influence the distance across phospholipid head groups [20]. Although the lipid composition was not actually measured in either PC or PG in this study, we can assume, based on the manufacturer's description (Sigma-Aldrich, St. Louis, MO, USA), that the unsaturation content is similar in both phospholipids, implying that both liposomal systems have an equivalent number of targets and identical accessibility of ROS/RNS for lipid peroxidation. Finally, Xia et al. (2015) also showed that only concentrations above $1 \%$ xanthophyll (canthaxanthin), with similar polar features as ASTA, diminish membrane fluidity (with head group region reorganization) in egg yolk PCL, compared to $\beta$-carotene and lutein [21]. The effective ASTA concentration used here was $25 \mu \mathrm{M}$, corresponding to $0.5 \%$ of total phospholipids in membranes.

In summary, observable differences in lipid peroxidation at different $\mathrm{pHs}$ could not be attributed to compacting effects on the head groups (becoming less accessible to pro-oxidants) or to significant changes in the water-lipid contact surface (an effect of changes in size or diameter) related to different phospholipid compositions or ASTA associations in our experimental liposome preparations.

\subsection{Astaxanthin Suppresses Cyt C/SIN-1-Induced Liposome Oxidation at $p H>7.4$}

Active mitochondria sustain a high membrane potential $(\Delta \Psi$ and $\Delta \mathrm{pH}$ during regular aerobic metabolism. Performing calibrations with the MitoTracker Red probe (Molecular Probes, Eugene, OR, USA), Porcelli et al. [22] estimated the $\mathrm{pHs}$ in the intermembrane space, cytosol, and mitochondrial matrix to be $6.88 \pm 0.09,7.59 \pm 0.01$, and $7.78 \pm 0.09$, respectively. On the other hand, key apoptotic events such as cyt c-activated caspase cascades and apoptotic Bcl-2/Bax pore formation involve the mild acidification of the cytosol down to $0.5 \mathrm{pH}$ units [23]. The activation of caspase pathway by 
native cyt $\mathrm{c}$ is optimum at $\mathrm{pH} 6.4$, but only $25 \%$ at $\mathrm{pH} 7.4$ [24]. In the present work, the $\mathrm{pH}$ range scrutinized (6.2 to 8.0) encompasses the hydrogen ion concentrations reported in active mitochondrial compartments during either regular metabolism or apoptosis.

Within the lipid bilayer, the intrinsic pKa of the phosphate group in PC is 0.8 as determined in monolayers using an $\mathrm{Hg}$-drop adsorption electrode [25]. The lack of the choline tetrammonium group moiety, turns PG more anionic than PC at pH 7.4, although no abrupt changes in the phosphate group $\mathrm{pKa}$ are expected to occur. Considering that the $\mathrm{pKa}$ of $\mathrm{PC}$ is 0.8 , no significant net charge variations in either PCL or 10\% PCPGLs would be observed in the experimental $\mathrm{pH}$ range studied here. The most significant effect of $\mathrm{pH}$ on lipid peroxidation is long known to occur in the presence of catalytic ferrous / ferric ions due to the production of hydroxyl radicals $\left(\mathrm{HO}^{\bullet}\right)$ involving $\mathrm{Fe}(\mathrm{OH})_{2}$ and $\mathrm{Fe}(\mathrm{OH})_{3}$ interconversion (Fenton reaction).

The $\mathrm{pH}$ is crucial to attain effective oxidizing/nitr(osyl)ating conditions to trigger peroxidation of lipid systems. Although SIN-1 decomposition generates $\mathrm{O}_{2}{ }^{\bullet-}$ and $\mathrm{NO} \mathrm{O}^{\bullet}$ radicals which decay to the oxidizing/nitrifying agent $\mathrm{ONOO}^{-}$[26], the latter species does not seem to be directly involved in cyt c modifications under our experimental conditions. Although iron-induced lipid peroxidation is enhanced at decreasing extracellular $\mathrm{pH}$ values [27], our data show that both TBARS and MDA levels were diminished at pHs 6.2 and 6.8 as compared to pHs 7.4 and 8.0 (Figures 3 and 4). This finding suggests a role for "free" ferrous ions in the initiation of lipid peroxidation upon SIN-1/cyt c treatment. Conformational changes surrounding the Met 80 ligand (sixth coordination site) of cyt $\mathrm{c}$ and its replacement by a Lys residue (probably Lys ${ }_{79}$ ) has an apparent pKa of approximately 9.0 [17]. Therefore, one can assume that no more than a $10 \%$ increase in cyt c-peroxidase activity would be expected from a $\mathrm{pH}$ increase within the $\mathrm{pH} 6.2-8.0$ range.

In summary, the $\mathrm{pH}$ range studied here is in accordance with the proton concentration in active mitochondrial compartments, thus favoring the initiation of apoptosis. Higher $\mathrm{pH}$ does not significantly enhance the peroxidase activation of cyt $\mathrm{c}$ or induce net membrane charges in either PCL (zwitterionic) or 10\% PCPGLs (anionic). Despite refuting the participation of peroxynitrite from SIN-1 decomposition here, the same array of ROS/RNS subsequently induce lipid peroxidation of PCL and $10 \%$ PCPGL. Thus, one can assume that any $\mathrm{pH}$-dependent changes in lipid peroxidation in liposomal systems would be solely caused by the varying scavenging properties of ASTA within membranes, for ASTA's effects on membrane physicochemical properties are also not plausible.

\subsection{Mechanism of Astaxanthin-Protective Role against Liposome Peroxidation}

ASTA usually achieves higher thermodynamic stability in lipid bilayers by allowing its carboxyl and hydroxyl polar groups to interact with the hydrophilic head groups of phospholipids on the opposite sides of the membrane, thus assuming a 'vertical' orientation across the lipid bilayer [28]. However, at a concentration of $25 \mu \mathrm{M}(0.5 \%$ compared to total lipid content in both PCL and PCPGL), no significant structural modifications induced by the association of ASTA with liposomes would cause significant changes in membrane fluidity and so effectively altering membrane permeabilization for water-soluble, pro-oxidative agents such as $\mathrm{H}_{2} \mathrm{O}_{2}$ and $\mathrm{NO}^{\bullet}$.

Noteworthy is that El-Agamey et al [29] used pulse radiolysis in an aqueous, $2 \%$ argon-saturated $(v / v)$ Triton $\mathrm{X}-100$ solution to study the effects of $\mathrm{pH}$ on ASTA anion radicals (ASTA ${ }^{\bullet-}$ ) derived from the scavenging activity of ASTA [29]. The pKa value of the ASTAH ${ }^{\bullet} /$ ASTA $^{\bullet-}$ pair was found to be 10.62 in a micelle-aqueous solution. This suggests that no significant fraction of ASTA ${ }^{\bullet-}$ is expected at $\mathrm{pH} 6.2(<0.004 \%)$ or $\mathrm{pH} 8.0(<0.3 \%)$. Considering the pKa value of 10.62 and, thereby, the predominant protonated form of ASTAH ${ }^{\bullet}$ radical, we can exclude the hypothesis that electrostatic repulsions of ASTA $^{\bullet-}$ radicals within membranes could interfere with the propagation and termination steps of lipid peroxidation in PC or 10\% PCPGLs.

Cis,trans-isomerization of the ASTA polyene chain is also a pH-dependent feature of carotenoid chemistry. Liu \& Osawa (2007) reported that 9-cis-ASTA exhibits higher antioxidant activity than the all-trans isomer in vitro [30]. However, while the fraction of 9-cis-ASTA isomers increases under heat 
and acidic conditions [31], ASTA displayed less inhibitory activity toward lipid peroxidation under more acidic conditions (pHs 6.2 and 6.8), as measured by either TBARS or MDA levels. Nevertheless, the percentage of cis-trans interconversion cannot explain the substantial antioxidant protection that ASTA elicits in PCL or PCPGLs from $37 \%$ to $45 \%$, at different pHs.

Previous work has shown that ASTA preferentially blocks lipid peroxidation at the polar membrane surface of egg yolk PC liposomes [28]. ASTA directly intercepts $\mathrm{O}_{2}{ }^{\bullet-}, \mathrm{NO}_{2}{ }^{\bullet}$, and $\mathrm{HO}^{\bullet}$ radicals, produced from SIN-1 decomposition in addition to lipoperoxyl (LOO ${ }^{\bullet}$ ) and lipoxyl $\left(\mathrm{LO}^{\bullet}\right)$ radicals formed during propagation of lipid peroxidation. Another relevant feature of ASTA chemistry is the formation of hydrogen bonds involving $\mathrm{C}=\mathrm{O}$ and $-\mathrm{OH}$ groups attached to its $\beta$-ionone rings. Intermolecular hydrogen bonds are normally formed between the two terminal polar rings of ASTA (hydroxyl-keto groups) and the polar phospholipid head groups, but there is also evidence that ASTA forms intramolecular hydrogen bonds between those same polar head groups [28]. The formation of intramolecular hydrogen-bonded five-membered rings increase the hydrophobicity of ASTA and favor its insertion into the hydrophobic core of the lipid bilayer. Such a translocation of the terminal rings of ASTA should be advantageous for scavenging lipid-soluble radicals such as $\mathrm{LOO}^{\bullet}$ and $\mathrm{LO}^{\bullet}$. In fact, ASTA is able to trap radicals by its conjugated polyene chain at the hydrophobic core of membranes, and in both hydrophobic core and water-lipid interfaces by its terminal ring moiety, based on switches between intramolecular hydrogen bonds and those formed with the polar groups of phospholipids [28]. Another hypothesis is that $\mathrm{pH}$ could influence the water-lipid surface/hydrophobic core distribution of ASTA molecules by keto-enol tautomerism. As an enolate, a negatively charged molecule, ASTA could not insert into the hydrophobic core of membranes to perform its scavenging properties. The calculation of the pKa of the enol/enolate form of ASTA is approximately 9.5 [32].

Therefore, among all the $\mathrm{pH}$-dependent factors investigated here, tautomerism of the ASTA keto-enol forms, $\mathrm{pH}$-dependent dissociation to its enolate form, and the formation of hydrogen bonds (intramolecular or with polar groups of phospholipids) are apparently the most plausible aspects of the complex chemistry of ASTA that could explain its varying antioxidant activity against mitochondrial-like redox conditions.

\section{Materials and Methods}

\subsection{Chemicals}

All chemicals used were obtained from Sigma-Aldrich Brasil Ltda. (São Paulo, Brazil) and the liquid chromatography grade solvents-n-hexane, chloroform, methanol and ethanol-purchased from Merck Co. (Darmstadt, Germany).

\subsection{Astaxanthin Stock Solutions}

Stock solutions of ASTA $(10 \mathrm{mM})$ were prepared in chloroform, of which the concentrations were determined by spectrophotometry $\left(\varepsilon=101 \mathrm{~L} \cdot \mathrm{mol}^{-1} \cdot \mathrm{cm}^{-1}\right.$ at $\left.486 \mathrm{~nm}\right)$. Stock solutions were stored at $-80^{\circ} \mathrm{C}$ and protected from light to avoid oxidation.

\subsection{Preparation of Unilamellar Liposomes}

Egg-yolk PC and egg-yolk PG were selected to prepare liposomes once: (i) PC and PG abound in biological membranes; (ii) egg-yolk phospholipids are polyunsaturated fatty acids (PUFAs)-rich, which are prone to suffer oxidative damage; and (iii) PC and PG have distinct net charges under different $\mathrm{pH}$ conditions thereby able to affect protein-lipid interactions in liposome systems. Astaxanthin stock solutions were previously mixed with phospholipid solutions in chloroform to reach a final carotenoid:phospholipid ratio of $0.5 \%$ mol ASTA, in order to avoid carotenoid aggregation in the formed liposomes. Final concentration of ASTA in liposomes was $25 \mu \mathrm{M}$. Control liposomes lack ASTA. Three liposome systems were prepared:

- $5 \mathrm{mM}$ phosphatidylcholine (5 $\mathrm{mM}$ PCL); 
- $4.76 \mathrm{mM}$ phosphatidylcholine + $0.24 \mathrm{mM}$ phpsphatidylglycerol (PCPGL 5\%); and

- $4.54 \mathrm{mM}$ phosphatidylcholine $+0.46 \mathrm{mM}$ phpsphatidylglycerol (PCPGL 10\%).

Chloroform was subsequently removed by flushing $\mathrm{N}_{2}$ in a round-bottom flask adapted to a rotavapor apparatus working at a low speed and moderate heating $\left(\leq 37^{\circ} \mathrm{C}\right)$ to allow the formation of a homogeneous dried ASTA: phospholipid film. The lipid-carotenoid film was stored overnight in the dark under vacuum to eliminate traces of solvent.

Multilamellar liposomes were prepared by vortexing volumes of $50 \mathrm{mM}$ phosphate buffer ( $\mathrm{pH}$ 6.2, $6.8,7.4$, or 8.0) added to the lipid film for $5 \mathrm{~min}$, at room temperature. Finally, unilamellar liposomes were prepared by extrusion through $100 \mu \mathrm{m}$-pore polycarbonate membranes (MilliPore, Burlington, MA, USA), at $37^{\circ} \mathrm{C}$, in a mini-extruder device (Avanti Polar Lipids, Inc., Alabaster, AL, USA). Through 15 passes, we obtained clean, transparent and homogeneous unilamellar PCL or PCPGL suspensions and immediately used them in the experiments. Usual sonication was avoided to prepare our liposomal systems here, since it precludes the release of $\mathrm{Ti}^{3+}$ ions into the reaction milieu (from the sonicator tip), which would artifactually induce lipid oxidation in liposomes during procedures.

\subsection{Dynamic Light Scattering Studies}

Dynamic light scattering (DLS) was used to determine the average liposome size in a Malvern Zetasizer Nano-series equipment (Malvern Instruments Zen 600, Malvern, UK). Aliquots from PC, PCPG5\% e PCPG10\% (with or without ASTA) stock solutions were diluted to a final concentration of $1 \mathrm{mM}$ total phospholipid in $50 \mathrm{mM}$ phosphate buffer, $\mathrm{pH} 7.4$, at $25^{\circ} \mathrm{C}$. To check ASTA incorporation into liposome membranes laser with $\lambda=590 \mathrm{~nm}$ was used.

\subsection{Oxidative/Nitrative Conditions for Liposome Peroxidation}

Five oxidative/nitrative different conditions (plus control) were studied to initiate lipid peroxidation in the normally aerated liposomal systems, in an attempt to mimic the regular redox environment in mitochondria. A $150 \mu \mathrm{M}$ 3-morpholinosydnonimine (SIN-1) solution was used as a biphasic generator of $\mathrm{O}_{2}{ }^{\bullet-}$ and $\mathrm{NO}^{\bullet}$ radicals, and presumably peroxynitrite $\left(\mathrm{ONOO}^{-}\right)$as the $\mathrm{O}_{2}{ }^{\bullet-} /$ $\mathrm{NO}^{\bullet}$ annihilation second order constant is $\sim 10^{10} \mathrm{M}^{-1} \mathrm{~s}^{-1}$. Spermin NONOate $(150 \mu \mathrm{M})$ was used as a single $\mathrm{NO}^{\bullet}$ donor. Based on previous studies, an equimolar $(150 \mu \mathrm{M})$ mixture of SIN-1 and cyt c better reproduces the redox scenario of the mitochondrial apoptotic condition [16]. All experiments and control tests were performed in $50 \mathrm{mM}$ phosphate buffer at various $\mathrm{pHs}, 37^{\circ} \mathrm{C}$, for $82 \mathrm{~min}$, considering the SIN-1 decay kinetics with $t_{1 / 2}=2900 \mathrm{~s}$. The oxidative/nitrative conditions (final concentrations in $1 \mathrm{~mL}$ ) are presented below:

- $5 \mathrm{mM}$ PCL/PCPGL $+50 \mathrm{mM}$ tPi (pH 6.2-8);

- $5 \mathrm{mM}$ PCL/PCPGL + $50 \mathrm{mM}$ tPi (pH 6.2-8) + $150 \mu \mathrm{M} \mathrm{SIN-1;}$

- $5 \mathrm{mM}$ PCL/PCPGL + $50 \mathrm{mM}$ tPi (pH 6.2-8) + $150 \mu \mathrm{M} \mathrm{SIN-1}+150 \mu \mathrm{M}$ cyt c;

- $5 \mathrm{mM}$ PCL/PCPGL + $50 \mathrm{mM}$ tPi (pH 6.2-8) + $150 \mu \mathrm{M} \mathrm{KO}_{2}$;

- $5 \mathrm{mM}$ PCL/PCPGL + $50 \mathrm{mM} \mathrm{tPi} \mathrm{(pH} \mathrm{6.2-8)} \mathrm{+} 150 \mu \mathrm{M} \mathrm{SIN-1} \mathrm{+} \mathrm{0.6} \mathrm{U/mL} \mathrm{SOD;}$

- $5 \mathrm{mM}$ PCL/PCPGL + $50 \mathrm{mM}$ tPi (pH 6.2-8) + $150 \mu \mathrm{M} \mathrm{KO}_{2}+0.6 \mathrm{U} / \mathrm{mL}$ SOD;

where: $\mathrm{tPi}=$ phosphate buffer; $\mathrm{KO}_{2}=$ potassium superoxide; and $\mathrm{SOD}=$ superoxide dismutase.

\subsection{UV-Vis Spectrophotometry}

Absorption spectra were traced on a Varian Carry 50 Bio UV-VIS spectrophotometer (Agilent Technologies, Santa Clara, CA, USA) at $37^{\circ} \mathrm{C}$. The time course of the thermal decomposition of SIN-1 was followed at $290 \mathrm{~nm}$, and product formation at $275 \mathrm{~nm}$. Structural changes of cyt c during reactions with SIN-1 were accompanied by scanning the 390-430 $\mathrm{nm} \lambda_{\max }$ shifts of the cyt c Soret band, which indicates any possible disruption of the hexacoordinate form of the heme iron [33]. Different SIN-1:cyt c ratios were employed to analyze the extent of SIN-1-promoted oxidative/nitrating effects to the 
hemeprotein: (i) $50 \mu \mathrm{M}$ cyt c $+0 \mu \mathrm{M}$ SIN-1 (control); (ii) $5 \mu \mathrm{M}$ cyt c $+50 \mu \mathrm{M}$ SIN-1; (iii) $50 \mu \mathrm{M}$ cyt c + $50 \mu \mathrm{M}$ SIN-1; and (iv) $50 \mu \mathrm{M}$ cyt c $+100 \mu \mathrm{M}$ SIN-1. The spectral background noise, which compromises the detection of slight shifts in the $\lambda_{\max }$ of the Soret band, was taken into account by adjusting the spectral data to a smoothing Gaussian-function (390-430 nm), defined by the formula:

$$
\left.\mathrm{y}=\mathrm{y}_{0}+\left[\mathrm{A} /\left(\mathrm{w}(\pi / 2)^{1 / 2}\right)\right] \mathrm{e}^{-2 \cdot[(\mathrm{x}-\mathrm{xc}) 2 / \mathrm{w} 2]}\right),
$$

where: $\mathrm{A}$, is the area under curve; $\mathrm{w}$, the band width (data distribution); $\mathrm{y}_{0}$, the curve cut off (baseline); $\mathrm{R}^{2}$, the correlation index; and $\mathrm{xc}$, the curve peak (calculated $\lambda_{\max }$ ).

\subsection{Nitric Oxide Concentration}

The NO ${ }^{\bullet}$ concentration was measured by the fluorescence intensity $\left(\lambda_{\mathrm{exc}}=495 \mathrm{~nm} ; \lambda_{\mathrm{em}}=515 \mathrm{~nm}\right)$ of the probe 4-amino-5-methylamino-2' ${ }^{\prime} 7^{\prime}$-difluorofluorescein (DAF-FM; Molecular Probes ${ }^{\circledR}$, Fisher Scientific, Pittsburgh, PA, USA). The manufacturers state that the fluorescence quantum yield $\left(\phi_{\mathrm{F}}\right)$ of DAF-FM is c.a. 0.005 , which roughly increases 160 -fold after reacting with $\mathrm{NO}^{\bullet}\left(\phi_{\mathrm{F}} \sim 0.81\right)$ in a $\mathrm{pH}$-independent process, $\mathrm{pH}>5.5$.

\subsection{Indices of Lipid Peroxidation}

Lipid peroxidation was evaluated from the observed concentrations of thiobarbituric acid-reactive substances (TBARS; spectrophotometric assay) and malondialdehyde (MDA; HPLC-UV/VIS detection) in the spent liposome oxidation sample. The peroxidation reactions were stopped by adding $8 \mathrm{mM}$ butylated hydroxytoluene (BHT) dissolved in ethanol. TBARS detection was performed with $200 \mu \mathrm{L}$ of sample after incubation at $100{ }^{\circ} \mathrm{C}$ for 15 min with $500 \mu \mathrm{L}$ of $0.25 \%$ thiobarbituric acid in $0.25 \mathrm{M} \mathrm{HCl}$, and $2 \%$ Triton X-100. After reaching room temperature, absorbances were measured at $535 \mathrm{~nm}$ (blanks lack thiobarbituric acid) using 1,1',2,2'-tetraethoxypropane as a standard, which decomposes in water to form malondialdehyde (MDA) [34]. After cleaning procedures, $20 \mu \mathrm{L}$ of the solution were injected into a Shimadzu SCL 10A HPLC system provided with a fluorescence detector RF-10AXL (Shimadzu Group Co., Kyoto, Japan) and isocratically eluted through a $\mathrm{C}_{18}$-Bondapack $0.39 \times 30 \mathrm{~cm}$ column by a 65:35 mixture of $25 \mathrm{mM}$ phosphate buffer ( $\mathrm{pH}$ 6.5) and 30\% methanol. The integrated area under MDA peaks (retention time $\sim 12 \mathrm{~min}$ ) was used to calculate MDA concentration based on a standard curve identical to that traced for the TBARS assay [35]. TBARS and MDA values were presented as relative values (A.U.) compared to respective controls in Figures 3-5 and Tables 2 and 3.

\subsection{Statistical Analysis}

Data are presented as mean \pm standard deviation, $(x \pm \mathrm{SD}, n \geq 3)$ and the statistical analysis performed with the Student's $t$ test at significance level of 5\% (OriginPro 2016, 64-bit, Sr-2). Graphics were plotted with Excel 2016, Microsoft Office 365, and OriginPro 2016, 64-bit, Sr-2.

\section{Conclusions}

This work contributes to a better understanding of the complex biochemistry of ASTA related to the scavenging of relevant ROS/RNS under different $\mathrm{pHs}$ and lipid membrane compositions. As shown here, ASTA presents a modest antioxidant activity under acidic conditions when zwitterionic or anionic liposomes are peroxidized during our apoptosis-like SIN-1/cyt c model system. Overwhelming efficacy of ASTA was however testified here under physiological ( $\mathrm{pH} 7.4)$ and mild alkaline conditions ( $\mathrm{pH}$ 8.0) in both PC and PG liposomal systems. The tautomerism of ASTA keto-enol forms (pH-dependent enolate formation) and the formation of hydrogen bonds (intramolecular or with polar groups of phospholipids) imply different antioxidant activities and thus better explain its antioxidant behavior. Once mitochondrial metabolism involves variations in $\Delta \Psi$ and $\Delta \mathrm{pH}$, we hypothesize that ASTA is endowed with $\mathrm{pH}$-dependent antioxidant/antiapoptotic properties in respiring mitochondria. 
Supplementary Materials: The following are available online at http:/ / www.mdpi.com/1660-3397/16/4/126/s1, Figure S1: (a) Decomposition mechanism of SIN-1 in aerated aqueous solution; (b) UV-Vis spectra of SIN-1 decomposition, for $90 \mathrm{~min}$, in $50 \mathrm{mM}$ phosphate buffer, $\mathrm{pH}$ 7.4, Figure S2: Fluorescence of the NO॰ sensitive probe DAF-FM in the presence of PCPGL, in $50 \mathrm{mM}$ phosphate buffer, $\mathrm{pH}$ 7.4, Figure S3: UV-Vis spectra of cyt c treated with spermine NONOate. Insert: fluorescence of DAF-FM as a function of the decomposition of spermine NONOate in $50 \mathrm{mM}$ phosphate buffer, pH 7.4, Table S1: Gaussian function parameters applied for the cyt c spectrum in the presence of SIN-1 and PCL, in $50 \mathrm{mM}$ phosphate buffer, $\mathrm{pH}$ 7.4, for 10 and $20 \mathrm{~min}$.

Acknowledgments: The authors are indebted to the former under graduate students and technicians of our laboratory for practical support during experiments. The authors are also thankful to Iseli L. Nantes, Tatiana Prieto, and Priscila A. Faria, from Federal University of ABC, UFABC, Santo Andre, SP, Brazil for very pertinent discussion and suggestions that significantly improved the quality of this study. This work was supported by the Fundação de Amparo à Pesquisa do Estado de São Paulo (FAPESP; BPE fellowship 2017/06032-2), by Conselho Nacional de Desenvolvimento Científico e Tecnológico (Bolsa Produtividade em Pesquisa, Nivel 2, 304663/2015-8, CNPq, Brazil), and by Programa Iberoamericano de Ciencia y Tecnología para el Desarollo (CYTED; Red iberoamericana para el estudio de nuevos carotenoides bioactivos como ingredientes de alimentos; 112RT0445, Spain).

Author Contributions: C.M.M. was responsible for all experiments during her undergraduate studies in Biological Sciences, Cruzeiro do Sul University, Sao Paulo, SP, Brazil, under my supervision M.P.B., and finished this study as a Ph.D. student at the Department of Biochemistry, University of Sao Paulo, SP, Brazil, under E.J.H.B. supervision. K.H.M.C. and T.G. performed the malondialdehyde-HPLC assays with C.M.M. at P.C. laboratory, also at the Department of Biochemistry, University of Sao Paulo, SP, Brazil. M.P.B., E.J.H.B., and P.C. participated in the experimental design and the full revision of the paper. Finally, M.P.B. conceived the study, obtained resources and financial support for the project, coordinated the experimental activities, analyzed the data, and together with E.J.H.B., wrote and corrected the initial draft, and further version of the manuscript.

Conflicts of Interest: The authors declare that there is no conflict of interest regarding the publication of this paper. Results of the present study do not constitute endorsement of any product by the authors or any private corporation or governmental agency.

\section{References}

1. Milani, A.; Basirnejad, M.; Shahbazi, S.; Bolhassani, A. Carotenoids: Biochemistry, pharmacology and treatment. Br. J. Pharmacol. 2017, 174, 1290-1324. [CrossRef] [PubMed]

2. Young, A.J.; Lowe, G.M. Antioxidant and prooxidant properties of carotenoids. Arch. Biochem. Biophys. 2001, 385, 20-27. [CrossRef] [PubMed]

3. Polotow, T.G.; Poppe, S.C.; Vardaris, C.V.; Ganini, D.; Guariroba, M.; Mattei, R.; Mattei, R.; Hatanaka, E.; Martins, M.F.; Bondan, E.F.; et al. Redox status and neuro inflammation indexes in cerebellum and motor cortex of wistar rats supplemented with natural sources of omega-3 fatty acids and astaxanthin: Fish Oil, Krill Oil, and Algal Biomass. Mar. Drugs 2015, 13, 6117-6137. [CrossRef] [PubMed]

4. Yuan, J.P.; Peng, J.; Yin, K.; Wang, J.H. Potential health-promoting effects of astaxanthin: A high-value carotenoid mostly from microalgae. Mol. Nutr. Food Res. 2011, 55, 150-165. [CrossRef] [PubMed]

5. Guerin, M.; Huntley, M.E.; Olaizola, M. Haematococcus astaxanthin: Applications for human health and nutrition. Trends Biotechnol. 2003, 21, 210-216. [CrossRef]

6. Kuroki, T.; Ikeda, S.; Okada, T.; Maoka, T.; Kitamura, A.; Sugimoto, M.; Kume, S. Astaxanthin ameliorates heat stress-induced impairment of blastocyst development in vitro: Astaxanthin colocalization with and action on mitochondria. J. Assist. Reprod. Genet. 2013, 30, 623-631. [CrossRef] [PubMed]

7. Bolin, A.P.; Macedo, R.C.; Marin, D.P.; Barros, M.P.; Otton, R. Astaxanthin prevents in vitro auto-oxidative injury in human lymphocytes. Cell Biol. Toxicol. 2010, 26, 457-467. [CrossRef] [PubMed]

8. Kavitha, K.; Kowshik, J.; Kishore, T.K.; Baba, A.B.; Nagini, S. Astaxanthin inhibits NF-kB and Wnt/ $\beta$-catenin signaling pathways via inactivation of Erk/MAPK and PI3K/Akt to induce intrinsic apoptosis in a hamster model of oral cancer. Biochim. Biophys. Acta 2013, 1830, 4433-4444. [CrossRef] [PubMed]

9. Kim, J.H.; Choi, W.; Lee, J.H.; Jeon, S.J.; Choi, Y.H.; Kim, B.W.; Chang, H.I.; Nam, S.W. Astaxanthin inhibits $\mathrm{H}_{2} \mathrm{O}_{2}$-mediated apoptotic cell death in mouse neural progenitor cells via modulation of P38 and MEK signaling pathways. J. Microbiol. Biotechnol. 2009, 19, 1355-1363. [CrossRef] [PubMed]

10. Jiang, X.; Wang, X. Cytochrome c-mediated apoptosis. Ann. Rev. Biochem. 2004, 73, 87-106. [CrossRef] [PubMed]

11. Byungki, J.; Sanghwa, H. Biochemical properties of cytochrome c nitrated by peroxynitrite. Biochimie 2006, 88, 53-58. [CrossRef] 
12. Kawai, C.; Ferreira, J.C.; Baptista, M.S.; Nantes, I.L. Not only oxidation of cardiolipin affects the affinity of cytochrome c for lipid bilayers. J. Phys. Chem. 2014, 118, 11863-11872. [CrossRef] [PubMed]

13. Sharpe, M.A.; Cooper, C.E. Reaction of nitric oxide with mitochondrial cytochrome c: A novel mechanism for the formation of nitroxyl anion and peroxide. Biochem. J. 1998, 332, 9-19. [CrossRef] [PubMed]

14. Schonhoff, M.C.; Gaston, B.; Mannick, J.B. Nitrosylation of cytochrome c during apoptosis. J. Biol. Chem. 2003, 278, 18265-18270. [CrossRef] [PubMed]

15. Wolf, A.M.; Asoh, S.; Hiranuma, H.; Ohsawa, I.; Iio, K.; Satou, A.; Ishikura, M.; Ohta, S. Astaxanthin protects mitochondrial redox state and functional integrity against oxidative stress. J. Nutr. Biochem. 2010, 21, 381-389. [CrossRef] [PubMed]

16. Mano, C.M.; Barros, M.P.; Faria, P.A.; Prieto, T.; Dyszy, F.H.; Nascimento, O.R.; Nantes, I.L.; Bechara, E.J. Superoxide radical protects liposome-contained cytochrome c against oxidative damage promoted by peroxynitrite and free radicals. Free Radic. Biol. Med. 2009, 47, 841-849. [CrossRef] [PubMed]

17. Rajagopal, B.S.; Edzuma, A.N.; Hough, M.A.; Blundell, K.L.I.M.; Kagan, V.E.; Kapralov, A.A.; Fraser, L.A.; Butt, J.N.; Silkstone, G.G.; Wilson, M.T.; et al. The hydrogen-peroxide-induced radical behavior in human cytochrome c-phospholipid complexes: Implications for the enhanced pro-apoptotic activity of the G41S mutant. Biochem. J. 2013, 456, 441-452. [CrossRef] [PubMed]

18. Cassina, A.M.; Hodara, R.; Souza, J.M.; Thomson, L.; Castro, L.; Ischiropoulos, H.; Freeman, B.A.; Radi, R. Cytochrome c nitration by peroxynitrite. J. Biol. Chem. 2000, 275, 21409-21415. [CrossRef] [PubMed]

19. Roy, B.; Guha, P.; Bhattarai, R.; Nahak, P.; Karmakar, G.; Chettri, P.; Panda, A.K. Influence of lipid composition, $\mathrm{pH}$, and temperature on physicochemical properties of liposomes with curcumin as model drug. J. Oleo Sci. 2016, 65, 399-411. [CrossRef] [PubMed]

20. Pan, J.; Heberle, F.A.; Tristram-Nagle, S.; Szymanski, M.; Koepfinger, M.; Katsaras, J.; Nučerka, N. Molecular structures of fluid phase phosphatidylglycerol bilayers as determined by small angle neutron and X-ray scattering. Biochim. Biophys. Acta 2012, 1818, 2135-2148. [CrossRef] [PubMed]

21. Xia, S.; Tan, C.; Zhang, Y.; Abbas, S.; Feng, B.; Zhang, X.; Qin, F. Modulating effect of lipid bilayer-carotenoid interactions on the property of liposome encapsulation. Colloids Surf. B Biointerfaces 2015, 128, 172-180. [CrossRef] [PubMed]

22. Porcelli, A.M.; Ghelli, A.; Zanna, C.; Pinton, P.; Rizzuto, R.; Rugolo, M. pH difference across the outer mitochondrial membrane measured with a green fluorescent protein mutant. Biochem. Biophys. Res. Commun. 2005, 326, 799-804. [CrossRef] [PubMed]

23. Matsuyama, S.; Llopi, J.; Deveraux, Q.; Tsien, R.; Reed, J. Changes in intramitochondrial and cytosolic pH: Early events that modulate caspase activation during apoptosis. Nat. Cell Biol. 2000, 2, 318-325. [CrossRef] [PubMed]

24. Matsuyama, S.; Reed, J.C. Mitochondria-dependent apoptosis and cellular pH regulation. Cell Death Differ. 2000, 7, 1155-1165. [CrossRef] [PubMed]

25. Moncelli, M.R.; Becucci, L.; Guidelli, R. The intrinsic pKa values for phosphatidylcholine, phosphatidylethanolamine, and phosphatidylserine in monolayers deposited on mercury electrodes. Biophys. J. 1994, 66, 1969-1980. [CrossRef]

26. Fiuza, B.; Subelzú, N.; Calcerrada, P.; Straliotto, M.R.; Piacenza, L.; Cassina, A.; Rocha, J.B.; Radi, R.; de Bem, A.F.; Peluffo, G. Impact of SIN-1-derived peroxynitrite flux on endothelial cell redox homeostasis and bioenergetics: Protective role of diphenyl diselenide via induction of peroxiredoxins. Free Radic. Res. 2015, 49, 122-132. [CrossRef] [PubMed]

27. Schafer, F.Q.; Buettner, G.R. Acidic pH amplifies iron-mediated lipid peroxidation in cells. Free Radic. Biol. Med. 2000, 28, 1175-1181. [CrossRef]

28. Goto, S.; Kogure, K.; Abe, K.; Kimata, Y.; Kitahama, K.; Yamashita, E.; Terada, H. Efficient radical trapping at the surface and inside the phospholipid membrane is responsible for highly potent antiperoxidative activity of the carotenoid astaxanthin. Biochim. Biophys. Acta 2001, 1512, 251-258. [CrossRef]

29. El-Agamey, A.; Edge, R.; Navaratnam, S.; Land, E.J.; Truscott, T.G. Carotenoid radical anions and their protonated derivatives. Org. Lett. 2006, 8, 4255-4258. [CrossRef] [PubMed]

30. Liu, X.; Osawa, T. Cis astaxanthin and especially 9-cis astaxanthin exhibits a higher antioxidant activity in vitro compared to the all-trans isomer. Biochem. Biophys. Res. Commun. 2002, 357, 187-193. [CrossRef] [PubMed] 
31. Mortensen, A.; Skibsted, L.H. Kinetics and mechanism of the primary steps of degradation of carotenoids by acid in homogeneous solution. J. Agric. Food Chem. 2000, 48, 279-286. [CrossRef] [PubMed]

32. Begum, S.; Cianci, M.; Durbeej, B.; Falklöf, O.; Hädener, A.; Helliwell, J.R.; Helliwell, M.; Regan, A.C.; Watt, C.l.F. On the origin and variation of colors in lobster carapace. Phys. Chem. Chem. Phys. 2015, 17, 16723-16732. [CrossRef] [PubMed]

33. Nantes, I.L.; Faljoni-Alário, A.; Nascimento, O.R.; Bandy, B.; Gatti, R.; Bechara, E.J.H. Modifications in heme iron of free and vesicle bound cytochrome c by tert-butyl hydroperoxide: A magnetic circular dichroism and electron paramagnetic resonance investigation. Free Radic. Biol. Med. 2000, 28, 786-796. [CrossRef]

34. Fraga, C.G.; Leibovitz, B.E.; Tappel, A.L. Lipid peroxidation measured as thiobarbituric acid-reactive substances in tissue slices: Characterization and comparison with homogenates and microsomes. Free Radic. Biol. Med. 1988, 4, 155-161. [CrossRef]

35. Domijan, A.M.; Ralić, J.; Radić-Brkanac, S.; Rumora, L.; Žanić-Grubišić, T. Quantification of malondialdehyde by HPLC-FL_Application to various biological samples. Biomed. Chromatogr. BMC 2015, 29, 41-46. [CrossRef] [PubMed]

(C) 2018 by the authors. Licensee MDPI, Basel, Switzerland. This article is an open access article distributed under the terms and conditions of the Creative Commons Attribution (CC BY) license (http:/ / creativecommons.org/licenses/by/4.0/). 